\title{
AN ALGEBRAIC CLASSIFICATION OF NONCOMPACT 2-MANIFOLDS
}

\author{
BY \\ MARTIN E. GOLDMAN( ${ }^{(1)}$
}

0. Introduction. We prove the following:

THEOREM. A surface $S$ is determined, up to homeomorphism, if the following are known:

(1) the vector space $H_{c}^{1}\left(S, Z_{2}\right)$,

(2) the Boolean ring $H_{e}^{0}\left(S, Z_{2}\right)$,

(3) the cup products

$$
\begin{gathered}
\cup: H_{c}^{1}\left(S, Z_{2}\right) \otimes H_{c}^{1}\left(S, Z_{2}\right) \rightarrow H_{c}^{2}\left(S, Z_{2}\right), \\
\cup: H^{1}\left(S, Z_{2}\right) \otimes H_{c}^{1}\left(S, Z_{2}\right) \rightarrow H_{c}^{2}\left(S, Z_{2}\right), \\
\cup: H_{e}^{0}\left(S, Z_{2}\right) \otimes H^{1}\left(S, Z_{2}\right) \rightarrow H_{e}^{1}\left(S, Z_{2}\right) .
\end{gathered}
$$

By a surface we mean a connected separable 2-manifold without border. $H^{q}\left(S, Z_{2}\right)$ and $H_{c}^{q}\left(S, Z_{2}\right)$ denote, respectively, the standard and compact support cohomology groups. $H_{e}^{q}\left(S, Z_{2}\right)$ is the cohomology group of the cochains of $S$ modulo the cochains of $S$ with compact support (see $\$ 1$ ).

For a closed surface the Theorem is a direct consequence of the Classification Theorem for Closed Surfaces. In this case $H_{e}^{0}\left(S, Z_{2}\right)=0$.

For an open surface the Theorem is an algebraic version of the Kerekjarto Classification Theorem ([9, p. 262] or [5, Chapter 5]). The Kerekjarto Theorem says that an open surface is topologically determined, modulo a compact subsurface, by its space of ends $e(S)$ [1, p. 82] and various subspaces of $e(S)$. In this paper we substitute for $e(S)$ the Boolean ring $H_{e}^{0}\left(S, Z_{2}\right)$.

In [2] we proved the Theorem by using (1) the Kerekjarto Theorem, (2) the Stone Representation Theorem [4, p. 168] which says that the compact totally disconnected space $e(S)$ is determined by the Boolean ring of continuous functions from $e(S)$ to $Z_{2}$, and (3) a result shown to me by W. S. Massey which says that this ring is isomorphic to $H_{e}^{0}\left(S, Z_{2}\right)$ (see Remark 1.7).

In this paper the Theorem is proven directly, without explicitly using ends. Essentially we follow the proof of the Kerekjarto Theorem as presented in [9, §4], changing the statements about $e(S)$ to statements about $H_{e}^{0}\left(S, Z_{2}\right)$. The above theorem occurs as Corollary 4.2 to Theorem 4.1 , which is a more specific result.

Received by the editors March 12, 1969.

(1) The research for parts of this paper was supported by NSF grant GP 8971. Other parts are based on the author's dissertation presented for the degree Doctor of Philosophy at Yale University, June 1967.

Copyright (C) 1971, American Mathematical Society 
Where the proof is very similar to the one in [9] we will just outline the steps, and the reader is referred to [9] for more detail.

This theorem has the following consequences:

THEOREM 7.1. If $S$ is an open surface and $i: S^{\prime} \subset S$ an open subsurface such that $i_{*}: H_{1}\left(S^{\prime}, Z_{2}\right) \rightarrow H_{1}\left(S, Z_{2}\right)$ is an isomorphism (singular homology theory), then $S$ and $S^{\prime}$ are homeomorphic.

THEOREM 8.1. Let $S$ be any open surface. If $T$ is a closed surface having Euler characteristic less than zero, and in the case where $S$ is nonorientable $T$ is also nonorientable, then $S$ is a covering space of $T$.

Throughout the paper, except in $\S 1$, only cohomology and homology theories with $Z_{2}$ coefficients will be used. This is necessary so we can use Poincaré Duality on nonorientable surfaces. Therefore when the coefficient group is omitted it will be understood to be $Z_{2}$.

The author is deeply indebted to W. S. Massey for suggesting the results obtained here and for the many suggestions he made.

1. Algebraic preliminaries. In this section we define the ring $H_{e}^{0}(X)=$ $H^{0}\left(C^{*}(X) / C_{c}^{*}(X)\right)$ and summarize some facts, mostly standard, about AlexanderSpanier cohomology theory ([10, pp. 306-323] or [7]). Except where specifically noted, this is the only cohomology theory we use.

Let $X$ be a locally compact Hausdorff space, and $R$ a commutative ring. The cochain groups (where we have already taken the quotient by the subgroup of cochains having empty support) and cohomology groups of $X$ with coefficients in $R$ are denoted by $C^{*}(X, R)$ and $H^{*}(X, R)$, respectively. The cochain groups and cohomology groups of $X$ with compact support are denoted by $C_{c}^{*}(X, R)$ and $H_{c}^{*}(X, R)$, respectively. If $i: Y \subset X$, the image of an element $u \in H^{q}(X, R)$ under the map $i^{*}: H^{q}(X, R) \rightarrow H^{q}(Y, R)$ will be denoted by $u / Y$; we will do likewise for cohomology with compact support.

1.1. Consider the cochain complex whose $q$ th group is $C^{q}(X, R) / C_{c}^{q}(X, R)$. The cohomology groups of this complex will be denoted by $H_{e}^{q}(X, R)$.

1.2. Since $C^{0}(X, R)$, the set of all functions from $X$ to $R$, is a ring under pointwise addition and multiplication of functions, so are $H^{0}(X, R), H_{c}^{0}(X, R)$, and $H_{e}^{0}(X, R)$. In fact, giving $R$ the discrete topology, $H^{0}(X, R)$ consists of the continuous functions from $X$ to $R ; H_{c}^{0}(X, R)$ consists of those continuous functions which are zero outside a compact subset of $X ; H_{e}^{0}(X, R)$ is the ring of functions from $X$ to $R$ which are continuous outside a compact subset of $X$ modulo those which are zero outside a compact subset.

1.3. In the usual way [10, p. 315], [7, Chapter 7] there are cup products

$$
\begin{gathered}
\cup: H_{c}^{p}(X, R) \otimes H_{c}^{q}(X, R) \rightarrow H_{c}^{p+q}(X, R), \\
\cup: H^{p}(X, R) \otimes H_{c}^{q}(X, R) \rightarrow H_{c}^{p+q}(X, R), \\
\cup: H_{e}^{p}(X, R) \otimes H^{q}(X, R) \rightarrow H_{e}^{p+q}(X, R) .
\end{gathered}
$$


1.4. The short exact sequence

$$
0 \rightarrow C_{c}^{q}(X, R) \rightarrow C^{q}(X, R) \rightarrow C^{q}(X, R) / C_{c}^{q}(X, R) \rightarrow 0
$$

induces a long exact sequence

$$
\cdots \longrightarrow H_{c}^{q}(X, R) \stackrel{i_{X}}{\longrightarrow} H^{q}(X, R) \rightarrow H_{e}^{q}(X, R) \longrightarrow H_{c}^{q+1}(X, R) \longrightarrow \cdots
$$

1.5. If $M$ is a manifold there is a natural equivalence between the AlexanderSpanier cohomology theories and the appropriate singular cohomology theories [10, p. 341]. Hence Poincaré Duality holds: If $\operatorname{dim} M=n$, then

$$
H^{q}\left(M, Z_{2}\right) \approx \operatorname{Hom}\left(H_{c}^{n-q}\left(M, Z_{2}\right), Z_{2}\right)
$$

where given $u \in H^{q}\left(M, Z_{2}\right)$ and $v \in H_{c}^{n-q}\left(M, Z_{2}\right), u(v)=u \cup v$. Likewise if $i_{M}: H_{c}^{q}\left(M, Z_{2}\right) \rightarrow H^{q}\left(M, Z_{2}\right)$ is the map of 1.4 , then using the above isomorphism, $i_{M}(x)(v)=i_{M}(x) \cup v=x \cup v$, where $x \in H^{q}\left(M, Z_{2}\right)$ and $v \in H_{c}^{n-q}\left(M, Z_{2}\right)$. Therefore

$$
\operatorname{ker} i_{M}=\left\{x \in H_{c}^{q}\left(M, Z_{2}\right) \mid x \cup H_{c}^{n-q}\left(M, Z_{2}\right)=0\right\} .
$$

1.6. If $i: A \subset X$ is a closed subset, the usual long exact sequence for the pair $(X, A)$ and the equivalence of the groups $H_{c}^{q}(X, A, R)$ and $H_{c}^{q}(X-A, R)$ [10, pp. 321-323] give us the long exact sequence

$$
\cdots \longrightarrow H_{c}^{q-1}(A, R) \stackrel{\delta}{\longrightarrow} H_{c}^{q}(X-A, R) \stackrel{\tau}{\longrightarrow} H_{c}^{q}(X, R) \stackrel{i^{*}}{\longrightarrow} H_{c}^{q}(A, R) \stackrel{\delta}{\longrightarrow} \cdots,
$$

where the map $\tau$ is induced by the "inclusion" $C_{c}^{q}(X-A, R) \subset C_{c}^{q}(X, R)[7$, Chapter 1].

If $r \in H^{1}(X, R), s \in H_{c}^{1}(X-A, R)$, then

$$
\tau((r / X-A) \cup s)=r \cup \tau(s) ;
$$

this is easily verified by looking at cochains.

1.7. Let $\left\{U_{\lambda}, \lambda \in \Lambda\right\}$ be the collection of subsets of $X$ having compact complement. The $U_{\lambda}$ are ordered by inclusion. The following result was noticed by W. S. Massey.

Lemma. For every $\lambda$ there is a map $\varphi: H^{q}\left(U_{\lambda}, R\right) \rightarrow H_{e}^{q}(X, R)$ and with these maps and the maps $i^{*}: H^{q}\left(U_{\lambda}, R\right) \rightarrow H^{q}\left(U_{\lambda^{\prime}}, R\right)$ induced by inclusions $U_{\lambda^{\prime}} \subset U_{\lambda}$, $H_{e}^{q}(X, R)$ is isomorphic to the direct limit of the groups $H^{q}\left(U_{\lambda}, R\right)$.

Proof. The inclusion $U_{\lambda} \subset X$ induces an epimorphism $C^{q}(X, R) \rightarrow C^{q}\left(U_{\lambda}, R\right)$. Denote its kernel by $C^{q}\left(X, U_{\lambda}, R\right)$. A simple verification shows that $C^{q}\left(X, U_{\lambda}, R\right) \subset$ $C_{c}^{q}(X, R)$ and in fact

$$
\sum_{\lambda} C^{q}\left(X, U_{\lambda}, R\right)=C_{c}^{q}(X, R)
$$

(where we are not taking the direct sum). Thus we have maps

$$
C^{q}\left(U_{\lambda}, R\right)=\frac{C^{q}(X, R)}{C^{q}\left(X, U_{\lambda}, R\right)} \rightarrow \frac{C^{q}(X, R)}{C_{c}^{q}(X, R)},
$$


and these maps induce an isomorphism

$$
\operatorname{dir} \lim _{\lambda} C^{q}\left(U_{\lambda}, R\right) \cong \frac{C^{q}(X, R)}{\sum C^{q}\left(X, U_{\lambda}, R\right)} \rightarrow \frac{C^{q}(X, R) .}{C_{c}^{q}(X, R)} .
$$

Passing to cohomology gives the desired maps.

Remark. Suppose $X^{*}$ is a compact Hausdorff space, $X$ an open dense subset and every point of $X^{*}-X$ has a system of neighborhoods each of whose intersection with $X$ is connected. If $N$ is an open neighborhood of $X^{*}-X$ then, using 1.2 ,

$$
i^{*}: H^{0}(N) \rightarrow H^{0}(N \cap X)
$$

is an isomorphism. Taking the direct limit over all $N$ and using the lemma and [10, p. 317], we have $H^{0}\left(X^{*}-X, R\right) \approx H_{e}^{0}(X, R)$. Letting $X$ be an open surface $S$ and $X^{*}$ its endpoint compactification, this becomes $H^{\circ}(e(S), R) \approx H_{e}^{0}(S, R)$, as was stated in the introduction.

2. Geometric preliminaries. From now on all homology and cohomology groups will have coefficients in $Z_{2}$. Everything we do will be from a piecewise linear point of view.

By a surface we mean a connected separable 2-dimensional manifold without border. Any surface can be triangulated and given a differentiable structure [1, pp. 107 and 127]. A surface is closed if it is compact, open if noncompact. We will also speak of bordered surfaces.

2.1. Any compact surface $K$, with or without border, is homeomorphic to a sphere with $m$ handles and $n$ cross-caps added and a finite number of open disks removed. The reduced genus of $K, g(K)$ is defined, as in [9, p. 260], to be $m+\frac{1}{2} n$. If $S$ is an open surface we define its reduced genus to be $g(S)=\sup g(K), K$ is a compact bordered subsurface of $S$.

If $S$ is closed $g(S)=\frac{1}{2} \operatorname{rank} H_{c}^{1}(S)$.

If $S$ is open,

$$
g(S)=\frac{1}{2} \operatorname{rank}\left[H_{\mathrm{c}}^{1}(S) / \operatorname{ker} i_{S}\right]
$$

$\left[1\right.$, p. 71, Theorem 31G] where $i_{S}: H_{c}^{1}(S) \rightarrow H^{1}(S)$, (see 1.4).

$S$ is planar if $g(S)=0$, i.e. if $\operatorname{ker} i_{S}=H_{c}^{1}(S)$.

Remark. Under Poincaré Duality $H_{c}^{1}(S)$ is isomorphic to the usual homology group $H_{1}(S)$. The subgroup ker $i_{S}$ corresponds to the homology classes of "dividing cycles" [1, p. 66]. By 1.4 , the rank of ker $i_{S}$ is one less than the rank of $H_{e}^{0}(S)$, which is in turn the number of ends of $S$ (see Remark 1.7).

2.2. There are four orientability classes of open surfaces. $S$ may be orientable. If $S-K$ is nonorientable for every compact subset $K$, then $S$ is infinitely nonorientable. If for some $K, S-K$ is orientable, then $S$ is said to be of even or odd nonorientability according as $K$ contains an even or an odd number of cross-caps [9, pp. 261-262]. Note that if we cap each component of the boundary of $K$ with 
a disk, then $S$ is of even or odd nonorientability according as this closed surface is cobordant to a sphere or a projective plane.

2.3. Let $S$ be a surface and let $w \in H^{1}\left(S, Z_{2}\right)$ denote the first Stiefel-Whitney class, i.e. the unique element satisfying $v \cup w=v \cup v$ for all $v \in H_{c}^{1}\left(S, Z_{2}\right)$, this being simply an extension of Wu's formula to an open surface (see [2, Lemma 6.3]).

A subsurface (with or without border) $T$ of $S$ is orientable if $w / T=0$.

The four orientability classes can be distinguished using $w$ as follows (the proof [2, Lemma 9.5] is easy).

LEMMA. A surface $S$ is

(i) orientable iff $w=0$,

(ii) infinitely nonorientable iff $w$ is not in the image of $i_{S}: H_{c}^{1}\left(S, Z_{2}\right) \rightarrow H^{1}\left(S, Z_{2}\right)$,

(iii) of even or odd nonorientability iff $w=i_{S}\left(w^{\prime}\right)$ and $w^{\prime} \cup w^{\prime}=0$ or 1 , respectively. (It is easily verified that $w^{\prime} \cup w^{\prime}$ is independent of the choice of $w^{\prime}$.)

2.4. Let $S$ be an open surface. A compact bordered subsurface $K$ will be called a canonical subsurface if it has all of the following properties:

(i) the closure of each component $U$ of $S-K$ is noncompact and meets $K$ in exactly one simple closed curve, called $d(U)$.

(ii) every component of $S-K$ is either planar or of infinite reduced genus, and is either orientable or infinitely nonorientable.

Because of (i), $H^{\circ}(S-K)$ is naturally isomorphic to $H^{\circ}(\mathrm{Bd} K)$ : a generator of $H^{0}(S-K)$ corresponding to a component $U$ of $S-K$ (i.e. the function $f: S-K \rightarrow$ $Z_{2}$ which is 1 on $U$ and 0 elsewhere) goes into the generator of $H^{\circ}(\mathrm{Bd} K)$ corresponding to $d(U)$. We will call the latter generator by the name $d(U)$.

Because of (i), the map $\varphi: H^{0}(S-K) \rightarrow H_{e}^{0}(S)$ of 1.7 is a monomorphism, so we can consider $H^{\circ}(S-K)$ as a subring of $H_{e}^{0}(S)$. Again we will call the element of $H_{e}^{0}(S)$ corresponding to the component $U$ of $S-K$ by the name $d(U)$.

From the above two paragraphs we obtain a monomorphism $H^{\circ}(\mathrm{Bd} K) \rightarrow$ $H_{e}^{0}(S)$, taking $d(U)$ into $d(U)$.

2.5. Every open surface $S$ has a canonical exhaustion, i.e. a collection $K_{0}, K_{1}, \ldots$ of canonical subsurfaces of $S$ such that $S=\bigcup_{i=0}^{\infty} K_{i}$ and $K_{i} \subset \operatorname{Int} K_{i+1}$ [1, p. 61].

2.6. Figure 2.6 shows five compact surfaces-three spheres with one, two, and three holes, respectively, and a torus and projective plane, each with two holes. On each surface, each component of the boundary has been oriented, and a graph with labeled and oriented edges has been embedded.

An open surface $S$ can be obtained inductively from these five by taking $K_{0}$ to be the sphere with one hole. Given $K_{i}, K_{i+1}$ is obtained by attaching to each border component of $K_{i}$ a copy of any one of the five surfaces except the sphere with one hole. This copy is attached by its left hand border component so that orientations of the border components match and the vertices are identified to each other. In this way we obtain a canonical exhaustion of $S$. Conversely because every open 

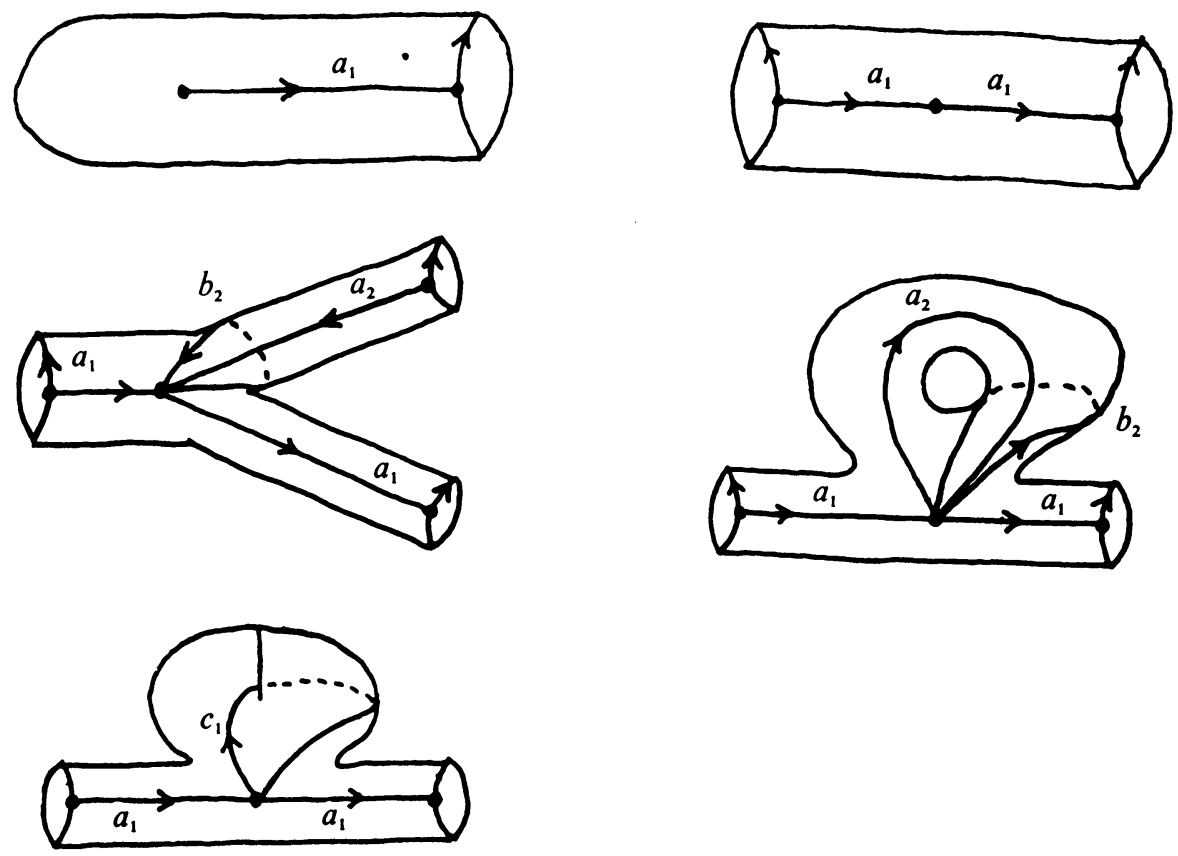

FIGURE 2.6

surface has a canonical exhaustion, it is easy to see that every open surface can be formed in this way [5, pp. 172-173].

In constructing $S$ we have also constructed a graph $G$ consisting of the $a_{i}, b_{i}$, and $c_{i}$. By [1, pp. 102-103] it is easily seen that $G$ provides a basis for $\pi_{1}(S)$ in the sense that $i_{*}: \pi_{1}(G) \rightarrow \pi_{1}(S)$ is an isomorphism, where $i: G \subset S$.

3. In this section we define two ideals $I_{S}$ and $J_{S}$ of $H_{e}^{0}\left(S, Z_{2}\right)$ having the property that a component $U$ of the complement of a canonical subsurface is infinitely nonorientable iff $d(U) \notin I_{S}$ and has infinite genus iff $d(U) \notin J_{S}$, where $d(U)$ is the element of $H_{e}^{0}(S)$ determined by $U(2.4)$.

3.1. LeMma. Let $K$ be a canonical subsurface of an open surface $S$ and $U$ a component of $S-K$.

(a) $U$ is orientable iff $w / U=0$, where $w$ is given by 2.3.

(b) $U$ is planar iff $y / U=0$ whenever $y \in H^{1}(S)$ has the property that $y \cup \operatorname{ker} i_{S}=0$.

Proof. (a) follows from 2.3.

For (b), suppose that $U$ is nonplanar. By 2.1, there is an element $x \in H_{c}^{1}(U)$ such that $x \notin \operatorname{ker} i_{U}$. By $1.5, \tau(x) \cup \operatorname{ker} i_{S}=0$, where $\tau: H_{c}^{1}(U) \rightarrow H_{c}^{1}(S)$, and hence $i_{S}(\tau(x)) \cup \operatorname{ker} i_{S}=0$. But $i_{S}(\tau(x)) / U=i_{U}(x)$ (verify on the cochain level) and hence $i_{S}(\tau(x)) / U \neq 0$.

Conversely, suppose now that $y \in H^{1}(S)$ and $y \cup \operatorname{ker} i_{S}=0$, but $y / U \neq 0$. By Poincaré Duality, $y / U \neq 0$ implies the existence of $x \in H_{c}^{1}(U)$ such that $(y / U) \cup x \neq$ 0 (see 1.5). 
We claim that since $y \cup \operatorname{ker} i_{S}=0$, it follows that $(y / U) \cup \operatorname{ker} i_{U}=0$. This will imply that $x \notin \operatorname{ker} i_{U}$ and hence that $U$ is nonplanar (2.1).

To prove the claim, we suppose $v \in \operatorname{ker} i_{U}$. It follows that $\tau(v) \in \operatorname{ker} i_{S}$ :

For $v \in \operatorname{ker} i_{U}$ implies that there is a $u \in C^{0}(U)$ such that $\delta u$ is a cocycle with compact support representing $v$. Since $\delta u$ has compact support in $U$, it is zero on a connected neighborhood of $\operatorname{Bd} U$. Hence $u$, as a function from $U$ to $Z_{2}$, is constant on that neighborhood. This implies that $u$ can be extended to a cochain $z \in C^{0}(S)$ (merely make it that constant on $S-U$ ) with $\delta z$ representing $\tau(v)$. Hence $i_{S}(\tau(v))=0$.

By 1.6, $\tau((y / U) \cup v)=y \cup \tau(v)$. But $y \cup \tau(v)=0$. Since $\tau: H^{2}(U) \rightarrow H^{2}(S)$ is an isomorphism, $(y / U) \cup v=0$, proving the claim.

3.2. Definition. Let

$$
\begin{aligned}
& I_{S}=\left\{x \in H_{e}^{0}(S) \mid x \cup w=0\right\}, \quad \text { where } w \text { is given by 2.3, } \\
& J_{S}=\left\{x \in H_{e}^{0}(S) \mid x \cup\left\{y \in H^{1}(S) \mid y \cup \operatorname{ker} i_{S}=0\right\}=0\right\}
\end{aligned}
$$

$I_{S}$ and $J_{S}$ are ideals of the ring $H_{e}^{\circ}(S)$.

3.3. Lemma. Let $K$ be a canonical subsurface of $S$, and $U$ a component of $S-K$.

(a) $U$ is infinitely nonorientable iff $d(U) \notin I_{S}$ (see 2.4).

(b) $U$ has infinite reduced genus iff $d(U) \notin J_{S}$.

Proof. For (a), suppose that $U$ is not infinitely nonorientable. There exists a compact subsurface $L$ of $S$ containing $K$ in its interior such that $U \cap(S-L)$ is orientable. By Lemma 3.1(a), $w / U \cap(S-L)=0$. But regarding $d(U) \in H^{0}(S-K)$,

$$
0=w / U \cap(S-L)=[d(U) / S-L] \cup(w / S-L) .
$$

By 1.7, this implies $d(U) \cup w=0$ and hence $d(U) \in I_{S}$. The reverse argument works equally well.

Using Lemma 3.1(b), the same argument gives a proof of the "if" part of (b).

Suppose now that $U$ has infinite reduced genus. We can find a canonical exhaustion $\left\{K_{i}\right\}$ of $S$ and compact bordered subsurfaces $\left\{C_{i}\right\}$ of $S$ such that $C_{i} \subset U \cap$ $\left(K_{i}-K_{i-1}\right)$ and $C_{i}$ has a handle or cross-cap on it. Associated with the handle or cross-cap on $C_{i}$, there is a cocycle $z_{i}$ with support in $C_{i}$ such that the element $\left[z_{i}\right]$ of $H_{c}^{1}(S)$ which $z_{i}$ represents is not in ker $i_{S}$. We can define the cocycle $z=\sum_{i=1}^{\infty} z_{i}$. $[z] \in H^{1}(S)$ and $d(U) \cup[z] \neq 0$. Suppose $[v] \in \operatorname{ker} i_{S}$. The support of $v$ is in some $K_{n}$ and $[z] \cup[v]=\sum_{i=1}^{n+1}\left[z_{i}\right] \cup[v]=0$ (see 1.5). Hence $d(U) \notin J_{S}$.

\section{The classification theorem.}

4.1. THEOREM. Two open surfaces $S$ and $S^{\prime}$ are homeomorphic iff they have the same genus and orientability class and there is a ring isomorphism $\theta: H_{e}^{0}\left(S^{\prime}, Z_{2}\right) \rightarrow$ $H_{e}^{0}\left(S, Z_{2}\right)$ taking $I_{S^{\prime}}$ onto $I_{S}, J_{S^{\prime}}$ onto $J_{S^{\prime}}$. 
This theorem will be proven in the next two sections. An immediate consequence is

4.2. Corollary. Two open surfaces are homeomorphic iff there are isomorphisms between $H_{c}^{1}\left(S, Z_{2}\right)$ and $H_{c}^{1}\left(S^{\prime}, Z_{2}\right), H^{1}\left(S, Z_{2}\right)$ and $H^{1}\left(S^{\prime}, Z_{2}\right)$, and a ring isomorphism between $H_{e}^{0}\left(S, Z_{2}\right)$ and $H_{e}^{0}\left(S^{\prime}, Z_{2}\right)$, these isomorphisms preserving all cup products (except that for $u \in H_{e}^{0}\left(S, Z_{2}\right), v \in H^{1}\left(S, Z_{2}\right)$ we need only require that $u \cup v=0$ iff the corresponding product on $S^{\prime}$ is zero).

Proof. See 4.1, 3.2, 1.5, 2.1, and 2.3.

REMARK. None of the three cup products can be omitted from the statement of the corollary. For example, consider the product between $H^{1}(S)$ and $H_{c}^{1}(S)$. Saying that it is preserved is equivalent to saying that the isomorphism between $H^{1}(S)$ and $H^{1}\left(S^{\prime}\right)$ is induced from the one between $H_{c}^{1}(S)$ and $H_{c}^{1}\left(S^{\prime}\right)$ (see 1.5). There are two infinitely nonorientable surfaces having two ends, both nonplanar. We can find isomorphisms between their cohomology groups satisfying all the conditions of the corollary except for preserving this product

4.3. REMARK. Under the correspondence between $\mathrm{H}_{e}^{0}(S)$ and the space of ends of $S$ referred to in the introduction, $I_{S}$ corresponds to the subspace of orientable ends, and $J_{S}$ to the subspace of planar ends. For more details see [2, $\$ 5,6$, and 10].

5. Inductive step. To prove Theorem 4.1 we will take canonical exhaustions of $S$ and $S^{\prime}$ and show, inductively, that they are homeomorphic. Here we develop the inductive step. The reader may look at subsections 5.1 and 5.4 for the main result. The proof is finished in $\S 6$.

5.1. Throughout $\S 5$ we will assume that $S$ and $S^{\prime}$ satisfy the hypothesis of Theorem 4.1. $K_{1}^{\prime}$ and $K_{2}^{\prime}$ will be canonical subsurfaces of $S^{\prime}, K_{1}^{\prime} \subset$ Int $K_{2}^{\prime}$. $K_{1}$ will be a canonical subsurface of $S$ and $f_{1}: K_{1} \rightarrow K_{1}^{\prime}$ a homeomorphism for which the diagram

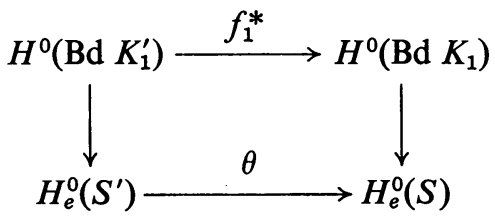

is commutative (the vertical maps are those described in 2.4).

5.2. Lemma. There is a canonical subsurface $K_{2}$ of $S$ containing $K_{1}$ in its interior and having the property that if $U$ and $U^{\prime}$ are corresponding components of $S-K_{1}$ and $S^{\prime}-K_{1}^{\prime}$, respectively, i.e. $f_{1}(\mathrm{Bd} U)=\mathrm{Bd} U^{\prime}$, then

(i) if $U^{\prime} \cap\left(K_{2}^{\prime}-K_{1}^{\prime}\right)$ is nonorientable then $U \cap\left(K_{2}-K_{1}\right)$ is nonorientable;

(ii) if $U^{\prime} \cap\left(K_{2}^{\prime}-K_{1}^{\prime}\right)$ has positive reduced genus, then $U \cap\left(K_{2}-K_{1}\right)$ has larger reduced genus.

Proof. Suppose $U^{\prime} \cap\left(K_{2}^{\prime}-K_{1}^{\prime}\right)$ is nonorientable. By 2.4(3), $U^{\prime}$ is infinitely nonorientable and hence, by $3.3(\mathrm{a}), d\left(U^{\prime}\right) \notin I_{S^{\prime}}$. By 5.1 , this says that $\theta\left(d\left(U^{\prime}\right)\right)=$ 
$d(U) \notin I_{S}$, i.e. $U$ is infinitely nonorientable. Hence, there exists a canonical subsurface $L$ containing $K_{1}$ in its interior such that $U \cap\left(L-K_{1}\right)$ is nonorientable.

Similarly, using 3.3(b), if $U^{\prime} \cap\left(K_{2}^{\prime}-K_{1}^{\prime}\right)$ has positive reduced genus, then $U$ has infinite reduced genus, and there is a canonical subsurface $N$ containing $K_{1}$ in its interior such that $g\left(U \cap\left(N-K_{1}\right)\right)>g\left(U^{\prime} \cap\left(K_{2}^{\prime}-K_{1}^{\prime}\right)\right)$.

Let $K_{2}$ be any canonical surface containing $K_{1}$ and all the $L$ 's and $N$ 's (one pair for each component $U$, if necessary) in its interior. $K_{2}$ satisfies the conclusion of the lemma.

5.3. LEMMA. $K_{1}$ is contained in the interior of a canonical subsurface $K_{2}$ such that

(i) regarding $H^{0}\left(S-K_{2}\right)$ and $H^{0}\left(S^{\prime}-K_{2}^{\prime}\right)$ as subgroups of $H_{e}^{0}(S)$ and $H_{e}^{0}\left(S^{\prime}\right)$ respectively (2.4),

$$
\theta\left(H^{0}\left(S^{\prime}-K_{2}^{\prime}\right)\right) \subset H^{0}\left(S-K_{2}\right),
$$

(ii) there is a partition $\mathscr{P}=\{P\}$ of the set of components of $S-K_{2}$ such that the members of $\mathscr{P}$ are in one-to-one correspondence with the components of $S^{\prime}-K_{2}^{\prime}$, and if $P \in \mathscr{P}$ corresponds to the component $U^{\prime}$, then

$$
\theta\left(d\left(U^{\prime}\right)\right)=\sum\{d(U) / U \in P\}
$$

(iii) all elements of the set $P$ of $\mathscr{P}$ corresponding to the component $U^{\prime}$ lie in the component of $S-K_{1}$ corresponding (under $f_{1} / \mathrm{Bd} K_{1}$ ) to the component of $S^{\prime}-K_{1}^{\prime}$ containing $U^{\prime}$.

Proof. For each component $U^{\prime}$ of $S^{\prime}-K_{2}^{\prime}$, there is a compact subset $L$ of $S$ such that (1.7)

$$
\theta\left(d\left(U^{\prime}\right)\right) \subset H^{0}(S-L) .
$$

Let $K_{2}$ be a canonical subsurface of $S$ containing all of the $L$ 's (one for each $U^{\prime}$ ). For each $U^{\prime}$,

and hence

$$
\theta\left(d\left(U^{\prime}\right)\right) \subset H^{0}\left(S-K_{2}\right)
$$

$$
\theta\left(H^{0}\left(S^{\prime}-K_{2}^{\prime}\right)\right) \subset H^{0}\left(S-K_{2}\right) .
$$

Let $\left\{U_{j} \mid j \in J\right\}$ and $\left\{U_{j}^{\prime} \mid j \in J^{\prime}\right\}$ be the components of $S-K_{2}$ and $S^{\prime}-K_{2}^{\prime}$, respectively. In the subring $H^{0}\left(S^{\prime}-K_{2}^{\prime}\right) \subset H_{e}^{0}\left(S^{\prime}\right)$,

$$
\begin{array}{ll}
d\left(U_{i}^{\prime}\right) \cdot d\left(U_{j}^{\prime}\right)=d\left(U_{i}^{\prime}\right), & \text { if } i=j \\
d\left(U_{i}^{\prime}\right) \cdot d\left(U_{j}^{\prime}\right)=0, & \text { if } i \neq j,
\end{array}
$$

and $\sum_{j \in J^{\prime}} d\left(U_{j}^{\prime}\right)=1$ (and of course the same equations are true in $H^{0}\left(S-K_{2}\right)$ when the primes are removed). Therefore since $\theta$ is a ring homomorphism taking 1 into 1 ,

$$
\begin{aligned}
\theta\left(d\left(U_{i}^{\prime}\right)\right) \cdot \theta\left(d\left(U_{j}^{\prime}\right)\right) & =\theta\left(d\left(U_{i}\right)\right), & & i=j \\
& =0, & & i \neq j
\end{aligned}
$$


and

$$
\sum_{j \in J^{\prime}} \theta\left(d\left(U_{j}^{\prime}\right)\right)=1
$$

Since the $d\left(U_{j}\right)$ are a basis for the vector space $H^{0}\left(S-K_{2}\right)$, we can write

$$
\theta\left(d\left(U_{i}^{\prime}\right)\right)=\sum_{t \in J} r_{i t} d\left(U_{t}\right), \quad i \in J^{\prime}, \quad r_{i t}=0 \text { or } 1,
$$

and (a) implies

$$
\begin{aligned}
r_{i t} \cdot r_{j t} & =r_{i t}, \quad i=j \\
& =0, \quad i \neq j .
\end{aligned}
$$

Letting $P_{i}=\left\{U_{t} / r_{i t}=1\right\}$ we obtain the desired partition; for by (c), if $r_{i t}=1$ then $r_{j t}=0, j \neq i$, and by (b), for each $t$, there must be at least one $i$ for which $r_{i t}=1$. This completes the proof of (ii).

For (iii), let $V^{\prime}$ be the component of $S^{\prime}-K^{\prime}$, containing $U^{\prime}$, and $V$ the component of $S-K$, corresponding to $V^{\prime}$, i.e. $d\left(V^{\prime}\right) \cdot d\left(U^{\prime}\right)=d\left(U^{\prime}\right)$ and $\theta\left(d\left(V^{\prime}\right)\right)=d(V)$. Since $\theta$ is a ring homomorphism and $\theta\left(d\left(U^{\prime}\right)\right)=\sum\{d(U) / U \in P\}$, it follows that

$$
d(V) \cdot\left(\sum\{d(U) / U \in P\}\right)=\sum\{d(U) / U \in P\} .
$$

Hence each $U \in P$ lies in $V$.

5.4. LEMMA. $K_{1}$ is contained in the interior of a canonical subsurface $K_{2}$, having the property that there exists a homeomorphism $f_{2}: K_{2} \rightarrow K_{2}^{\prime}$ extending $f_{1}$ and making the diagram

commutative.

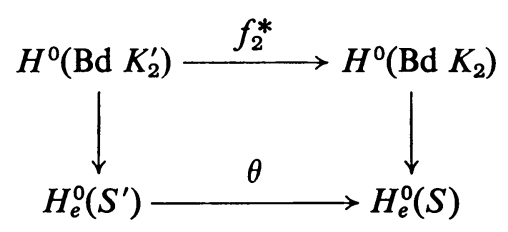

Proof. Let $K_{2}$ be a canonical subsurface containing the $K_{2}$ 's found in Lemmas 5.2 and 5.3. This $K_{2}$ will satisfy the conclusions of these two lemmas. Because of Lemma 5.2 we can diminish $K_{2}$, if necessary, so that each component of $K_{2}-K_{1}$ has the same reduced genus as the corresponding component of $K_{2}^{\prime}-K_{1}^{\prime}$ and is orientable iff the latter is. This can be done so that Lemma 5.3 still holds: take a collar of a suitable component of $\mathrm{Bd} K_{2}$ (i.e., one corresponding to a component of $S-K_{2}$ which is infinitely nonplanar or infinitely nonorientable, as is appropriate) lying in $K_{2}-K_{1}$ and move the excess handles and cross-caps into it; then remove this set from $K_{2}$.

Because of 5.3(iii) we can assume, by diminishing $K_{2}-K_{1}$, that each element of $\mathscr{P}=\left\{P_{j}\right\}$ of 5.3 contains exactly one component of $S-K_{2}$; this is done in the manner illustrated in Figure 5.4, where we suppose that originally $P_{1}=\left\{U_{1}, U_{2}, U_{3}\right\}$, 


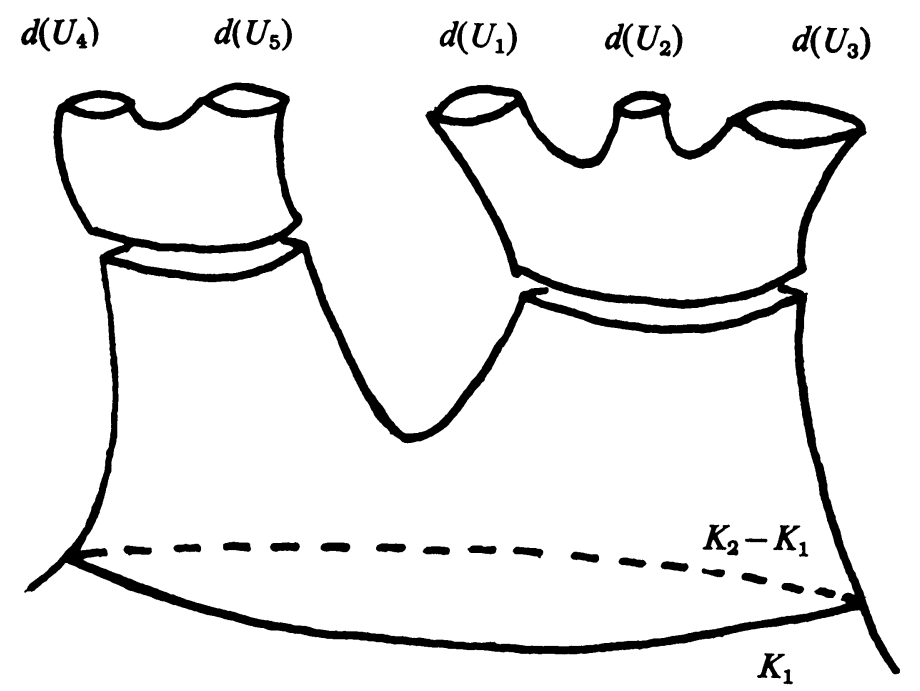

FIGURE 5.4

$P_{2}=\left\{U_{4}, U_{5}\right\}$. The reader wishing more detail is referred to $[9$, p. 265 , last two paragraphs].

Now for every component $U_{j}^{\prime}$ of $S^{\prime}-K_{2}^{\prime}$ the corresponding $P_{j}$ will consist of exactly one component $U_{j}$ of $S-K_{2}$ and, by 5.3(ii), $\theta\left(d\left(U_{j}^{\prime}\right)\right)=d\left(U_{j}\right)$.

Each component of $K_{2}-K_{1}$ now has the same reduced genus and number of boundary components as the corresponding component of $K_{2}^{\prime}-K_{1}^{\prime}$, and is orientable iff the latter is. Hence we can construct a homeomorphism of $\mathrm{Cl}\left(K_{2}-K_{1}\right)$ onto $\mathrm{Cl}\left(K_{2}^{\prime}-K_{1}^{\prime}\right)$ which agrees with $f_{1}$ on $\mathrm{Bd} K_{1}$ and which takes $d\left(U_{j}\right)$ onto $d\left(U_{j}^{\prime}\right), j \in J^{\prime}$. Thus, we have a homeomorphism $f_{2}: K_{2} \rightarrow K_{2}^{\prime}$ making the diagram in the statement of the lemma commutative.

6. Proof of Theorem 4.1. When taking a canonical exhaustion (2.5) $K_{0}, K_{1}$, $K_{2}, \ldots$ of a surface $S$ we may take $K_{0}$ to have only one boundary component. By 2.4(ii), if $S$ has finite reduced genus, then $K_{0}$ has the same reduced genus and is orientable iff $S$ is. If $S$ has infinite reduced genus and is of even or odd nonorientability, take $K_{0}$ to be, respectively, a Klein bottle or a projective plane with an open disk removed. In all other cases take $K_{0}$ to be a disk.

Let $\left\{K_{i}\right\}$ and $\left\{K_{i}^{\prime}\right\}$ be canonical exhaustions of $S$ and $S^{\prime}$, where $K_{0}$ and $K_{0}^{\prime}$ satisfy the conditions of the preceding paragraph. There is a homeomorphism $f_{0}: K_{0} \rightarrow K_{0}^{\prime}$ making, trivially, the diagram

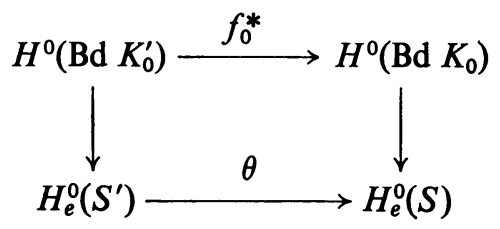

commutative, where, as usual, the vertical maps are from 2.4. 
We now make an inductive argument in the process of which we replace the exhaustions $\left\{K_{i}\right\}$ and $\left\{K_{i}^{\prime}\right\}$ by canonical exhaustions $\left\{L_{i}\right\}$ and $\left\{L_{i}^{\prime}\right\}$ of $S$ and $S^{\prime}$ determined in accordance with the following diagram. We begin with $L_{0}=K_{0}$ and $L_{0}^{\prime}=K_{0}^{\prime}$.

Suppose we already have $L_{i}, L_{i}^{\prime}$ and a homeomorphism $f_{i}: L_{i} \rightarrow L_{i}^{\prime}$ making the diagram

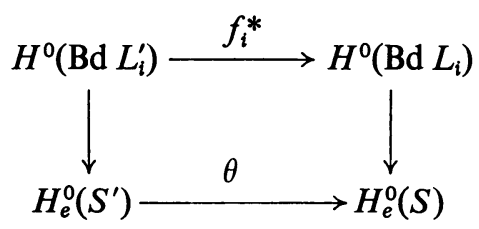

commutative. Furthermore if $i$ is even we will suppose that $K_{i} \subset L_{i}$ and if $i$ is odd, $K_{i}^{\prime} \subset L_{i}^{\prime}$. (This condition is imposed to insure that $\left\{L_{i}\right\}$ and $\left\{L_{i}^{\prime}\right\}$ encompass all of $S$ and $S^{\prime}$.)

Suppose that $i+1$ is odd. Pick a $K_{j}^{\prime}$ containing both $L_{i}^{\prime}$ and $K_{i+1}^{\prime}$ in its interior. Define $L_{i+1}^{\prime}$ to be $K_{j}^{\prime}$. By 5.4, there exists a canonical subsurface $L_{i+1}$, containing $L_{i}$ in its interior, and a homeomorphism $f_{i+1}: L_{i+1} \rightarrow L_{i+1}^{\prime}$ extending $f_{i}$ and making the diagram

commutative.

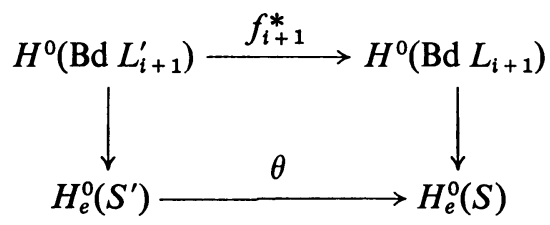

If $i+1$ is even, pick a $K_{j}$ containing both $L_{i}$ and $K_{i+1}$ in its interior. Define $L_{i+1}$ to be $K_{j}$. By 5.4 , there exists a canonical subsurface $L_{i+1}^{\prime}$ containing $L_{i}^{\prime}$ in its interior, and a homeomorphism $f_{i+1}: L_{i+1} \rightarrow L_{i+1}^{\prime}$ extending $f_{i}$ and making the preceding diagram commutative.

Thus we have exhaustions $\left\{L_{i}\right\}$ and $\left\{L_{i}^{\prime}\right\}$ and homeomorphisms $f_{i}: L_{i} \rightarrow L_{i}^{\prime}$ such that $f_{i} / L_{i-1}=f_{i-1}$. Hence there is a homeomorphism $f: S \rightarrow S^{\prime}$ (i.e. $f / L_{i}=f_{i}$ ).

7. Applications. In this and the next section we give some applications of Theorem 4.1. These will all be consequences of Theorem 7.1 below. Lemma 7.1 and Corollary 7.4 are due to W. S. Massey.

7.1. LemMA. Let $U$ be a noncompact, locally compact Hausdorff space and $U^{\prime}$ an open noncompact subset. Assume $U$ and $U^{\prime}$ are connected. If $(1.6) \tau: H_{c}^{1}\left(U^{\prime}\right) \rightarrow$ $H_{c}^{1}(U)$ is an isomorphism and $i^{*}: H^{1}(U) \rightarrow H^{1}\left(U^{\prime}\right)$ is a monomorphism, then $H_{e}^{0}(U)$ and $H_{e}^{0}\left(U^{\prime}\right)$ are isomorphic as rings. 
Proof. We have the following commutative diagram in which the rows are short exact sequences:

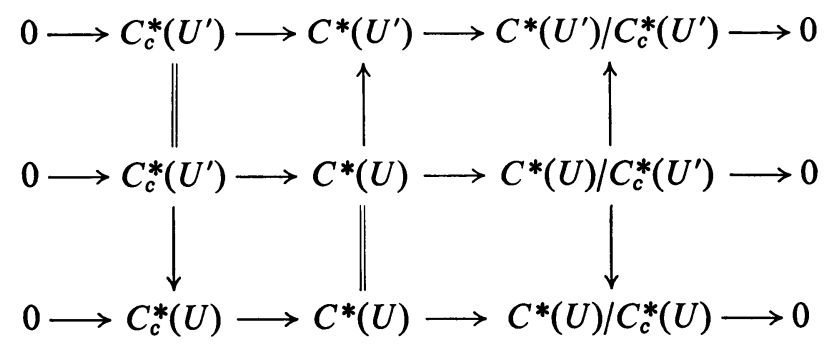

Passing to cohomology this yields the following commutative diagram in which the rows are long exact sequences:

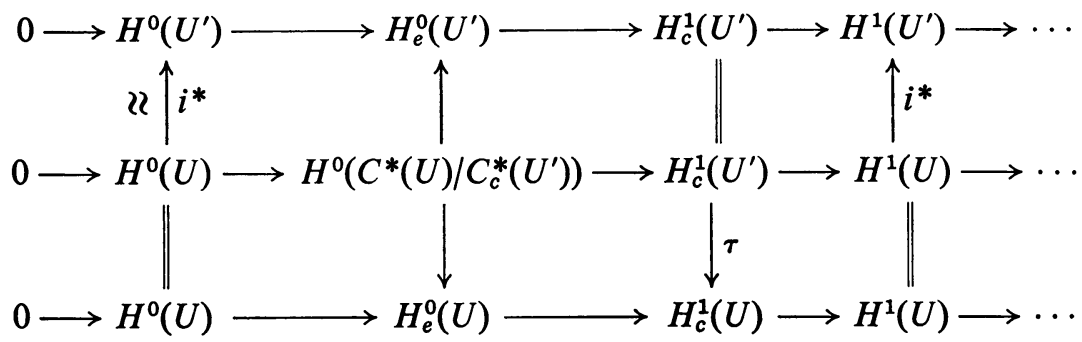

Note that in dimension zero all of the above maps are ring homomorphisms.

Under our hypotheses the 5-lemma states that $H_{e}^{0}\left(U^{\prime}\right) \approx H^{0}\left(C^{*}(U) / C_{c}^{*}\left(U^{\prime}\right)\right) \approx$ $H_{e}^{0}(U)$.

THEOREM. Let $S$ be an open surface and $i: S^{\prime} \subset S$ an open subsurface. If $i_{*}$ : $H_{1}\left(S^{\prime}, Z_{2}\right) \rightarrow H_{1}\left(S, Z_{2}\right)$ is an isomorphism in singular homology theory, then $S$ and $S^{\prime}$ are homeomorphic.

Proof. Using Poincaré Duality (1.5) we have the following commutative diagrams in which the vertical arrows are isomorphisms:
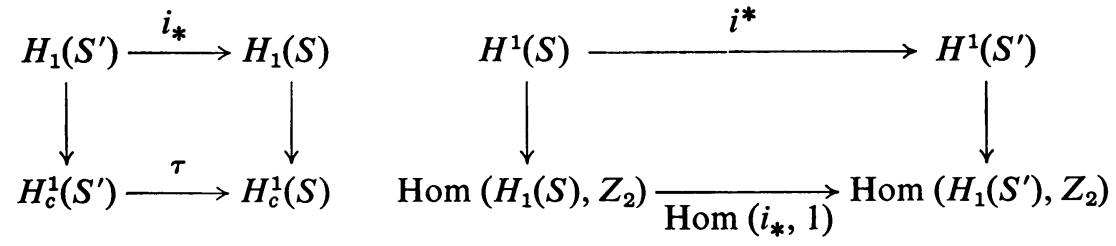

Therefore, if $i_{*}$ is an isomorphism, so are $\tau$ and $i^{*}$. By the preceding lemma, there is a ring isomorphism between $H_{e}^{0}(S)$ and $H_{e}^{0}\left(S^{\prime}\right)$. Cup products are preserved under the vertical maps in the diagram contained in the proof of that lemma. Hence by Corollary 4.2, $S$ and $S^{\prime}$ are homeomorphic.

7.2. Definition. Let $S$ be a triangulated surface and $G$ a subcomplex of the 1-skeleton. The thickening of $G$ in $S$ is the interior of the union of all closed simplices of the second barycentric subdivision of $S$ which meet $G$. 
CoROllary. Let $i: G \subset S$ be a graph such that $i_{*}: H_{1}(G) \rightarrow H_{1}(S)$ is an isomorphism, and let $N$ be a thickening of $G$ in $S$. Then $N \cong S$ (cf. [11, Lemma 2.1]).

Proof. Since $G$ is a deformation retract of $N, i_{*}: H_{1}(N) \rightarrow H_{1}(S)$ is an isomorphism. The result now follows from Theorem 7.1.

7.3. In 2.6 we showed how to construct a model of any open surface $S$. In addition we imbedded a graph $G$ in $S$, so that $i_{*}: \pi_{1}(G) \rightarrow \pi_{1}(S)$ was an isomorphism. By 7.2, $S$ is homeomorphic to a thickening of $G$. Referring to Figure 2.6, this says that any open surface can be built inductively from the five noncompact bordered surfaces shown in Figure 7.3 by joining copies of these surfaces along their borders in a manner similar to that described in 2.6 .
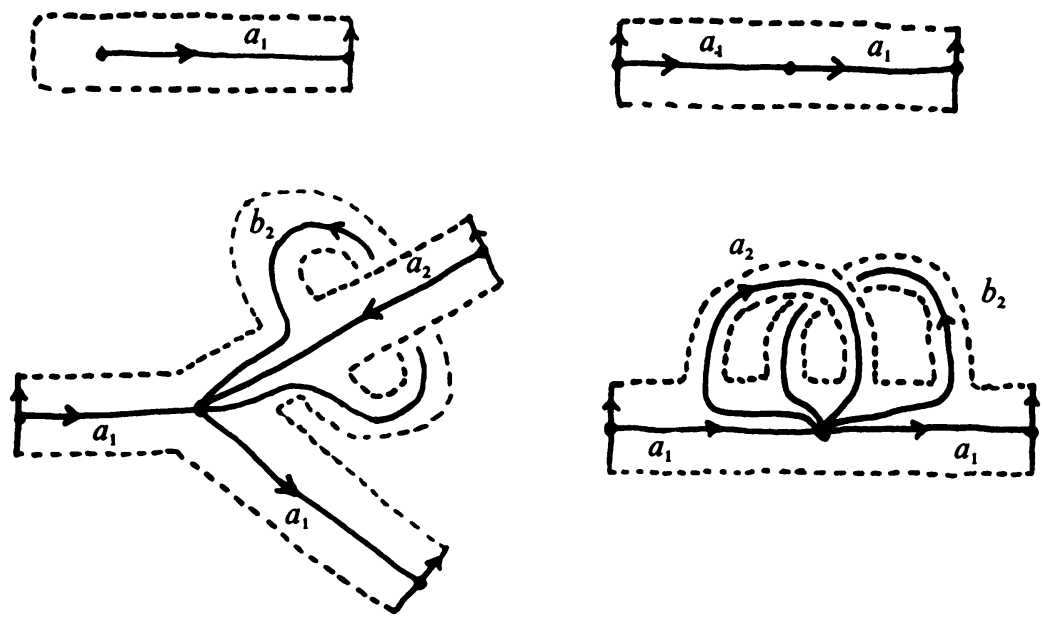

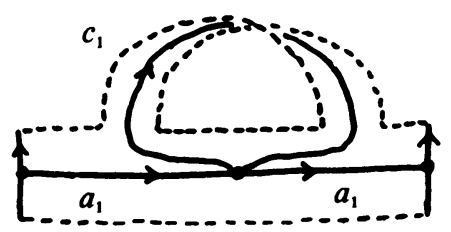

FIGURE 7.3

7.4. If $T^{\prime}$ is an open subsurface of a closed surface $T$ and $i_{*}: \pi_{1}\left(T^{\prime}\right) \rightarrow \pi_{1}(T)$ is a monomorphism, then there is obviously a one-to-one correspondence between covering spaces of $T^{\prime}$ and the covering spaces of $T$ corresponding to subgroups of im $i_{*}$. The following corollary of Theorem 7.1 says that corresponding covering spaces are homeomorphic.

COROLlARY. Let $S$ be an open surface, $T$ any surface, and $i: T^{\prime} \subset T$ a proper open subsurface. Choose a base point $o \in T^{\prime}$ and suppose that $i_{*}: \pi_{1}\left(T^{\prime}, o\right) \rightarrow \pi_{1}(T, o)$ is 
a monomorphism. Then there is a covering space $p: S \rightarrow T[8, \mathrm{p} .145]$ such that $\operatorname{im} p_{*} \subset \operatorname{im} i_{*}$ iff there is a covering space $p^{\prime}: S \rightarrow T^{\prime}$.

Proof. Let $p: S, \tilde{o} \rightarrow T$, $o$ be a covering space such that im $p_{*} \subset \operatorname{im} i_{*}$. Let $R$ be the component of $p^{-1}\left(T^{\prime}\right)$ containing $\tilde{o}$ and let $r=p / R$. Then $r: R \rightarrow T$ is the covering space corresponding to $i_{*}^{-1}\left(\operatorname{im} p_{*}\right)=\operatorname{im} r_{*}[8$, p. 177].

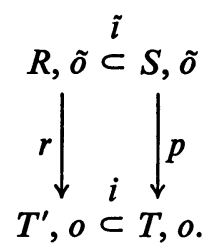

$\tilde{i}_{*}$ is an isomorphism. (It is a monomorphism because $r_{*}$ and $i_{*}$ are monomorphisms. It is an epimorphism because $\operatorname{im} p_{*} \subset \operatorname{im} i_{*}$ and the homotopy lifting property.) Hence by Theorem 7.1, $R$ and $S$ are homeomorphic.

Conversely if $r: R, \tilde{o}^{\prime} \rightarrow T^{\prime}, o$ is a covering space, let $p: S, \tilde{o} \rightarrow T$, $o$ be the covering space such that im $p_{*}=i_{*}\left(\operatorname{im} r_{*}\right), r: R, \tilde{o}^{\prime} \rightarrow T^{\prime}, o$ is equivalent, as a covering space, to the restriction of $p$ to the component of $p^{-1}(T)$ containing $\tilde{o}$. As in the preceding paragraph $R$ and $S$ are homeomorphic.

\section{Every open surface covers a closed one.}

8.1 THEOREM. Let $S$ be any open surface. If $T$ is a closed surface having reduced genus $>1$, i.e. $\chi(T)<0$, and in the case where $S$ is nonorientable $T$ is also nonorientable, then there is a covering space $p: S \rightarrow T$.

Proof. We will suppose that $T$ is nonorientable and $S$ is any surface. (The case where both $S$ and $T$ are orientable can be treated in a similar fashion.) A closed surface $T$ having $1<g(T) \leqq 2$ is covered by a closed surface having genus $>2$. Hence we may assume that $g(T)>2$.

Choose a base point $o \in T$. There are simple closed curves $a_{1}, b_{1}, a_{2}, b_{2}$, $c_{1}, \ldots, c_{k}(k \geqq 1)$ based at $o$ with the property that any two intersect only in the point $o$, and

$$
\pi_{1}(T, o)=\left(a_{1}, b_{1}, a_{2}, b_{2}, c_{1}, \ldots, c_{k} ; a_{1} b_{1} a_{1}^{-1} b_{1}^{-1} a_{2} b_{2} a_{2}^{-1} b_{2}^{-1} c_{1}^{2} \cdots c_{k}^{2}\right) .
$$

Let $G_{T}$ be the graph in $T$ having as its vertex $o$, and as its edges the curves $a_{1}, a_{2}, b_{2}$, and $c_{1}$. Figure 8.1a shows a neighborhood of $o$ in the thickening (7.2) of $G_{T}$ in $T$. Call this thickening $T^{\prime}$.

By the Frieheitssatz [6, p. 252] the subgroup of $\pi_{1}(T, o)$ generated by $a_{1}, a_{2}, b_{2}$, and $c_{1}$ is free on these generators. Hence $i_{*}: \pi_{1}\left(G_{T}, o\right) \rightarrow \pi_{1}(T, o)$ is a monomorphism. By 7.4, to prove the theorem it suffices to show that $S$ is a covering space of $T^{\prime}$. 


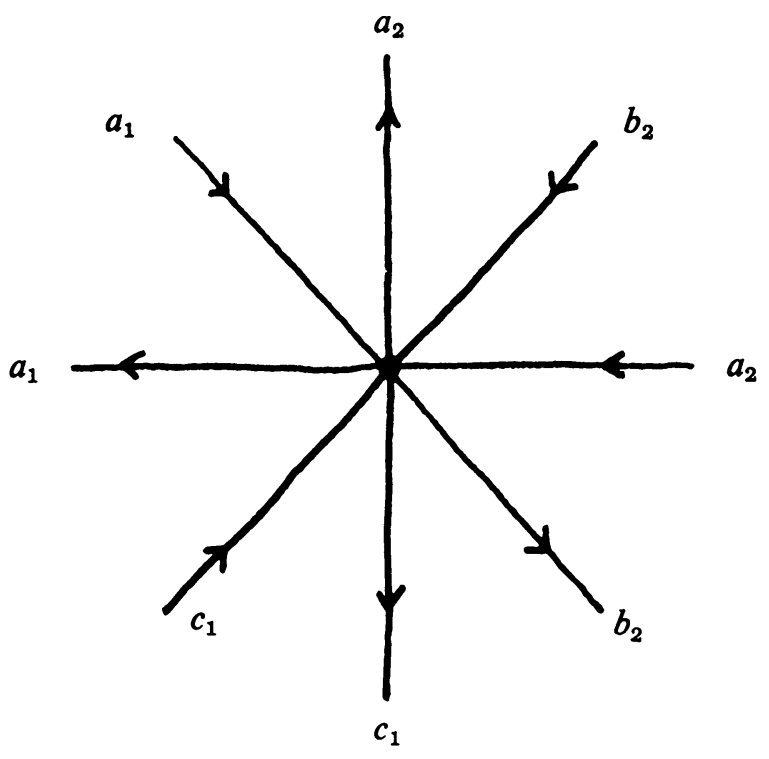

Figure 8.1a

Represent $S$ as the thickening of a graph $G_{S}$ as in 7.3. In describing $G_{S}$ in 7.3 we labeled and oriented its edges (Figure 7.3). This prescribes a local homeomorphism [8, p. 149] $p: G_{S} \rightarrow G_{T}$ taking an edge of $G_{S}$, minus its vertices, homeomorphically onto the corresponding edge of $G_{T}$, minus the vertex $o$. $G_{S}$ was imbedded in $S$ in just such a way as to insure that $p$ would extend to a local homeomorphism $P: S \rightarrow T^{\prime}$, taking the strip about an edge of $G_{S}$ homeomorphically onto the strip about the corresponding edge of $G_{T}$, except perhaps near the vertices.

The maps $p$ and $P$ look almost like covering maps. The problem is that a neighborhood of each vertex of $G_{S}$ should look like a neighborhood of $o$ in $T^{\prime}$, i.e. there should be some more edges present. So we add them and strips about them so that $P$ can be extended to a map of the thickening of the enlarged graph. The new edges are not loops so the enlarged surface is homeomorphic to $S$. Of course we now have to apply this process to the vertices we have just added. The entire procedure can be described as follows:

Let $q$ : $U \rightarrow T^{\prime}$ be the universal covering space. Pick $\tilde{o} \in U$ such that $q(\tilde{o})=o$. Removing a neighborhood $N$ of $\tilde{o}$ separates $U$ into 8 components, as shown in Figure $8.1 \mathrm{~b}$, where the edges are labeled so as to correspond to the edges they map into. In the preceding paragraph we attached at each vertex $v$ of $S$ a number of edges. For each edge, instead of attaching just that edge, attach the corresponding component of $U-N$, i.e. instead of attaching an edge $b_{2}$ directed away from $v$, attach the far right component of $U-N$ in Figure 8.1b. Extend $P$ to the component by setting it equal to $q$ there. Thus we obtain a new surface $S^{\prime}$ and a covering space $P^{\prime}: S^{\prime} \rightarrow T^{\prime}$. Each component of $U-N$ is the thickening of a tree 


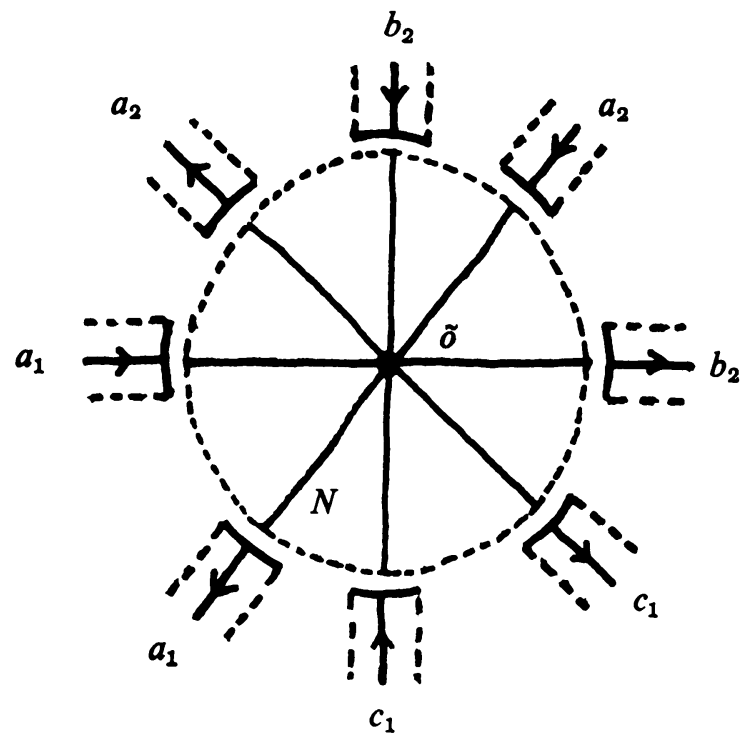

FIGURE 8.1b

[8, p. 194] and hence is a disk with part of its boundary. Therefore $S^{\prime}$ is homeomorphic to $S$ and we have found the required covering space $S \rightarrow T^{\prime}$

8.2. Remark. Theorem 8.1 covers the case when $\chi(T)<1$. If $\chi(T)=0, T$ is either a torus or a Klein bottle and it is not difficult to see that the only possible open surfaces covering $T$ are the plane and the annulus $[2, \S 16]$. If $\chi(T)>0, T$ is either a sphere or a projective plane, and hence there are no open surfaces covering $T$.

8.3. REMARK. If we require that the covering space $p: S \rightarrow T$ be regular, i.e. given any loop $c$ in $T$, either every lifting of $c$ is a loop or none is, the situation changes radically. It is not true that every open surface is a regular covering space of a closed surface. In fact using [3, p. 93, Satz II] it is easy to see that there are only nine open surfaces which can be regular covering spaces of a closed surface. In particular the space of ends of the surface must be homeomorphic to either a one point space, a two point space, or the Cantor set, and if the surface is planar, nonplanar but orientable, or nonorientable then all ends must be planar, nonplanar but orientable, or nonorientable, respectively. (For more detail see $[2, \S 15]$.)

\section{BIBLIOGRAPHY}

1. L. V. Ahlfors and L. Sario, Riemann surfaces, Princeton Math. Series, no. 26, Princeton Univ. Press, Princeton, N. J., 1960. MR 22 \#5729.

2. M. E. Goldman, Open surfaces and an algebraic study of ends, Thesis, Yale Univ., New Haven, Conn., 1967.

3. H. Hopf, Enden offener Räume und unendliche diskontinuierliche Gruppen, Comment. Math. Helv. 16 (1944), 81-100. MR 5, 272.

4. J. L. Kelley, General topology, Van Nostrand, Princeton, N. J., 1955. MR 16, 1136. 
5. B. Kerekjarto, Vorlesungen über Topologie. Vol. I, Springer, Berlin, 1923.

6. W. Magnus, A. Karrass and D. Solitar, Combinatorial group theory. Presentations of groups in terms of generators and relations, Pure and Appl. Math., vol. 13, Interscience, New York, 1966. MR 34 \#7617.

7. W. S. Massey, Notes on homology and cohomology theory, Mimeographed Notes, Dept. of Math., Yale Univ., New Haven, Conn., 1964.

8. - Algebraic topology. An introduction, Harcourt, Brace, and World, New York, 1967. MR 35 \#2271.

9. I. Richards, On the classification of noncompact surfaces, Trans. Amer. Math. Soc. 106 (1963), 259-269. MR 26 \#746.

10. E. H. Spanier, Algebraic topology, McGraw-Hill, New York, 1966. MR 35 \#1007.

11. J. H. C. Whitehead, The immersion of an open 3-manifold in euclidean 3-space, Proc. London Math. Soc. (3) 11 (1961), 81-90. MR 23 \#A2224.

RICE UNIVERSITY, Houston, TeXas 77001

City College of the City University of New York, NEW YoRK, NeW YoRK 10031 